\title{
ON CHANGES OF MEASURE IN STOCHASTIC VOLATILITY MODELS
}

\author{
BERNARD WONG AND C. C. HEYDE
}

Received 12 July 2006; Accepted 5 October 2006

Pricing in mathematical finance often involves taking expected values under different equivalent measures. Fundamentally, one needs to first ensure the existence of ELMM, which in turn requires that the stochastic exponential of the market price of risk process be a true martingale. In general, however, this condition can be hard to validate, especially in stochastic volatility models. This had led many researchers to "assume the condition away," even though the condition is not innocuous, and nonsensical results can occur if it is in fact not satisfied. We provide an applicable theorem to check the conditions for a general class of Markovian stochastic volatility models. As an example we will also provide a detailed analysis of the Stein and Stein and Heston stochastic volatility models.

Copyright (c) 2006 B. Wong and C. C. Heyde. This is an open access article distributed under the Creative Commons Attribution License, which permits unrestricted use, distribution, and reproduction in any medium, provided the original work is properly cited.

\section{Introduction}

In mathematical finance it is often assumed that a stochastic volatility model holds with dynamics under the risk-neutral measure $Q$ given by, for example,

$$
\begin{gathered}
d X(t)=\mu X(t) d t+X(t) \sqrt{V(t)}\left(\rho d W_{1}^{Q}(t)+\sqrt{1-\rho^{2}} d W_{2}^{Q}(t)\right), \\
d V(t)=\kappa(\theta-V(t)) d t+\sigma_{v} \sqrt{V(t)} d W_{1}^{Q}(t),
\end{gathered}
$$

with $W_{1}^{Q}(\cdot), W_{2}^{Q}(\cdot)$ Q-Brownian motions. We assume that $X(\cdot)$ is the stock price and $V(\cdot)$ is the state variable driving the volatility.

Given the stock price and savings account only, it is generally believed that there exist many different equivalent (local) martingale measures such that the discounted stock price is a (local) martingale. In particular, one question of importance is whether the conditions which are required for there to exist at least one ELMM are actually satisfied. This 
is important as the fundamental theorem of asset pricing (Delbaen and Schachermayer [7]) shows the equivalence between the existence of ELMMs and the economic condition of "no free lunch with vanishing risk." In the stochastic volatility model case however the unbounded nature of the volatility process often creates difficulties in trying to verify the technical conditions assumed by the theorems.

Given that ELMMs exist, a secondary question is whether it is actually an equivalent martingale measure. This has significance for pricing via expectation techniques, and can lead to pricing irregularities (Sin [30], Cox and Hobson [4]). A related problem is whether numeraire processes are true martingales, which allows the use of change of numeraire techniques (Geman et al. [14]), and is particularly important for consistent models of exchange rates (Delbaen and Schachermayer [9]).

In this paper we will investigate these martingale conditions for a general class of Markovian stochastic volatility models advocated by Hoffman et al. [19] and Frey [12]. This class of models moreover nests the popular models used in the literature, including the models of Hull and White [20], Heston [18], and Stein and Stein [31]. An applicable theorem relating to the validity of the measure transformations for a broad class of stochastic volatility models is presented, and also applied in detail to the Heston stochastic volatility model.

It is known that the (class of) equivalent martingale measures $Q$, if it exists, can be considered in terms of the Radon-Nikodym derivative

$$
\begin{aligned}
{\left.\frac{d Q}{d P}\right|_{T}}_{T}=L(T)=\exp \{ & -\left(\int_{0}^{T} \gamma_{1}(u) d W_{1}(u)+\int_{0}^{T} \gamma_{2}(u) d W_{2}(u)\right) \\
& \left.-\frac{1}{2}\left(\int_{0}^{T} \gamma_{1}^{2}(u) d u+\int_{0}^{T} \gamma_{2}^{2}(u) d u\right)\right\},
\end{aligned}
$$

where the $\gamma_{i}((\cdot)), i=1,2$, are the "market price of risk" processes corresponding to $W_{i}(\cdot)$, $i=1,2$.

As an illustration of what can go wrong when the existence assumptions are not checked, Rydberg [27] in an investigation in to the generalized hyperbolic diffusion models for stock prices (Rydberg [28], Bibby and Sørensen [2]) discovered that a check of the technical conditions for the existence of an ELMM reveals that no ELMM can exist for these models in general. Interestingly this adverse result is also present in other wellknown models. In particular we will show in Section 2 that the Stein and Stein [31] model also suffers the same problem.

Note that the results on existence of an ELMM, beside being of fundamental economic importance, are also very useful and important for statistical inference, especially for cases involving the joint dynamics of options and stocks (see, e.g., Chernov and Ghysels [3]). Furthermore they are also important when one wants to investigate numerical comparisons of hedging strategies (Heath et al. [15]). As a practical matter it may also serve as a reality check on a model that has been calibrated to market data.

It is somewhat surprising that, in all but the simplest of cases, these assumptions are often taken on trust. In general they are only verified when $\gamma(\cdot)$ is a constant, or at best, 
bounded. Unfortunately these represent very special cases and in fact most stochastic volatility models assume unbounded $V(\cdot)$ and $\gamma(\cdot)$.

Notice that since $L(\cdot)$ is a positive local martingale with $L(0)=1$, it is a supermartingale and is a true martingale if and only if

$$
E[L(T)]=1
$$

(cf. Karatzas and Shreve [22, Chapter 1]), and it is a required condition for Girsanov's theorem. Sufficient conditions for this include the Novikov condition and the Kazamaki condition. See, for example, Revuz and Yor [25, Chapter VIII] and Wong and Heyde [32].

This paper is structured as follows. In Section 2 we will show that the Stein and Stein [31] model allows for arbitrage opportunities, further highlighting the potential issues that can arise in stochastic volatility models. The Heston model will be considered in detail in Section 3, and we find that an intuitively reasonable restriction on the market price of risk parameter will ensure that the postulated ELMMs exist. The question of whether discounted stock prices are true martingales under the ELMM will also be addressed. The methodology and theorems derived in Section 3 will be extended in Section 4 to be applicable to a wide class of Markovian stochastic volatility models classified by Hoffman et al. [19].

Throughout this paper, we will be considering the time interval $[0, T]$ and probability space $(\Omega, \mathscr{F}, \mathbf{P})$, the filtration being generated by two-dimensional Brownian motion $\left\{W_{1}(\cdot), W_{2}(\cdot)\right\}$ augmented to satisfy the usual conditions.

\section{Arbitrage in the Stein and Stein model}

The Stein and Stein [31] model assumes real world dynamics (i.e., under the measure $P$ ) of

$$
\begin{aligned}
& d X(t)=\mu X(t) d t+X(t) U(t) d W_{1}(t), \\
& d U(t)=\kappa(\theta-U(t)) d t+\sigma_{v} d W_{2}(t),
\end{aligned}
$$

with $X(0), U(0)>0$, and parameters $\mu, \kappa, \theta, \sigma_{v}>0$ which for simplicity are assumed to be constants. Extensions to this model have been considered by Schöbel and Zhu [29], who also point out that the model does not include a reflecting barrier at 0 , nor deal with the absolute value $|U(\cdot)|$, points often claimed incorrectly in the literature (e.g., Stein and Stein [31], Ball and Roma [1]).

Denote $r$ as the constant risk-free rate with $\mu \neq r$. As we wish to arrive at an ELMM $Q$, we require a first market price of risk process $\gamma_{1}(\cdot)$ with

$$
\begin{aligned}
d X(t) & =\mu X(t) d t+X(t) U(t)\left(d W_{1}^{Q}(t)-\gamma_{1}(t) d t\right) \\
& =\left(\mu-U(t) \gamma_{1}(t)\right) X(t) d t+X(t) U(t) d W_{1}^{Q}(t),
\end{aligned}
$$

such that the Q-dynamics are

$$
d X(t)=r X(t) d t+X(t) U(t) d W_{1}^{Q}(t) .
$$


4 On changes of measure in stochastic volatility models

Hence on comparison with (2.1) a necessary condition is that for $t \in[0, T]$,

$$
\mu-U(t) \gamma_{1}(t)=r
$$

but as 0 lies in the interior of $U(\cdot)$ it is clear then that no ELMM can exist in general, and that this model will allow "immediate arbitrage" opportunities (Delbaen and Schachermayer [8]).

This type of simple check above has been surprisingly absent for many models in the literature. While the above calculations show quite clearly the problems for the Stein and Stein model, unfortunately the existence or otherwise of ELMMs is less clear for other models. An important caveat is that, even if we have

$$
P\left(\int_{0}^{T}\|\gamma(u)\|^{2} d u<\infty\right)=1
$$

it is not guaranteed that $L(\cdot)$ is a true martingale. Classical examples of this include the important Johnson and Helms [21] counterexample (see also Revuz and Yor [25, page 383] for additional discussion).

\section{Heston stochastic volatility model}

In this section we will provide a detailed analysis regarding the measure transformations in the Heston stochastic volatility model. Despite its documented econometric shortcomings (see, e.g., Chernov and Ghysels [3]) this model has enjoyed great popularity due to its relative simplicity and the availability of essentially closed form option price formulas for various derivative securities. The Heston model assumes real-world dynamics (i.e., under the measure $P$ ) of the form

$$
\begin{gathered}
d X(t)=\mu X(t) d t+X(t) \sqrt{V(t)}\left(\rho d W_{1}(t)+\sqrt{1-\rho^{2}} d W_{2}(t)\right) \\
d V(t)=\kappa(\theta-V(t)) d t+\sigma_{v} \sqrt{V(t)} d W_{1}(t)
\end{gathered}
$$

with $X(0), V(0)>0$, and parameters $\mu, \kappa, \theta, \rho, \sigma_{v}>0$ which for simplicity are assumed to be constants. From the theory of Bessel processes (cf. Revuz and Yor [25, Chapter XI]) we will impose the condition

$$
2 \kappa \theta \geq \sigma_{v}^{2}
$$

to ensure that volatility will be strictly positive in finite time.

A necessary condition for an ELMM to exist is

$$
\mu-r=\sqrt{V(t)}\left(\rho \gamma_{1}(t)+\sqrt{1-\rho^{2}} \gamma_{2}(t)\right)
$$

which ensures that discounted stock prices are local martingales. Following the work of Cox et al. [5], Heston [18] proposed the additional restriction (see also Dai and Singleton [6], Duffee [10] for a discussion in a related setting)

$$
\gamma_{1}(t)=\lambda \sqrt{V(t)}
$$


with $\lambda$ a constant. Notice that, provided that $P$ and $Q$ exist, we have

$$
\begin{aligned}
d V(t) & =\kappa(\theta-V(t)) d t+\sigma_{v} \sqrt{V(t)} d W(t) \\
& =\kappa^{*}\left(\theta^{*}-V(t)\right) d t+\sigma_{v} \sqrt{V(t)} d W^{Q}(t),
\end{aligned}
$$

and hence we maintain the same functional form under $Q$, with

$$
\kappa^{*}=\left(\kappa+\lambda \sigma_{v}\right), \quad \theta^{*}=\frac{\kappa \theta}{\left(\kappa+\lambda \sigma_{v}\right)} .
$$

On substitution in (3.3), noting that $V(\cdot)$ stays away from 0 and $\infty$ with probability one under our parameter restrictions (as verified by Feller's test for explosions, cf. Rogers and Williams [26, Section V]), we have as the second market price of risk process

$$
\gamma_{2}(t)=\frac{1}{\sqrt{1-\rho^{2}}}\left(\frac{\mu-r}{\sqrt{V(t)}}-\lambda \rho \sqrt{V(t)}\right)
$$

3.1. Existence of an ELMM. For the postulated ELMM to exist, we require the process $L(\cdot)$ to be a true martingale, where

$$
L(T)=L_{1}(T) L_{2}(T)
$$

with

$$
\begin{aligned}
& L_{1}(t)=\exp \{\left.-\int_{0}^{t} \lambda \sqrt{V(u)} d W_{1}(u)-\frac{1}{2} \int_{0}^{t}(\lambda \sqrt{V(u)})^{2} d u\right\}, \\
& L_{2}(t)=\exp \left\{-\int_{0}^{t} \frac{1}{\sqrt{1-\rho^{2}}}\left(\frac{\mu-r}{\sqrt{V(u)}}-\lambda \rho \sqrt{V(u)}\right) d W_{2}(u)\right. \\
&-\left.\frac{1}{2} \int_{0}^{t}\left(\frac{1}{\sqrt{1-\rho^{2}}}\left(\frac{\mu-r}{\sqrt{V(u)}}-\lambda \rho \sqrt{V(u)}\right)\right)^{2} D u\right\} .
\end{aligned}
$$

Furthermore, as $W_{2}(\cdot)$ and $V(\cdot)$ are independent, and $0<V(t)<\infty, t \leq T$, with probability 1 , by conditional expectations we have

$$
E[L(T)]=E\left[L_{1}(T)\right]
$$

Hence this is essentially just a one-dimensional problem for $L_{1}(\cdot)$. As it is a stochastic exponential it is a local martingale and a supermartingale, but due to the unbounded nature of $V(\cdot)$ it is not clear per se if it is a true martingale. 
6 On changes of measure in stochastic volatility models

Our solution to the aforementioned problem will make use of the following lemma regarding the existence of the bivariate Laplace transform of $\left(V(T), \int_{0}^{T} V(u) d u\right)$.

Lemma 3.1. For $\alpha \geq 0, \beta \geq-\kappa^{2} / 2 \sigma_{v}^{2}$,

$$
E\left[\exp \left\{-\alpha V(T)-\beta \int_{0}^{T} V(u) d u\right\}\right]<\infty
$$

Proof. For $\alpha \geq 0, \beta \geq 0$ the expectation can be calculated explicitly and is a direct consequence of results attributed to Feller [11] and Pitman and Yor [24] (see also Remark 3.2 below). For our applications to the Heston model however it is vital that $\beta<0$ be considered.

Consider a process $\{M(t)\}_{0 \leq t \leq T}$ with

$$
M(t)=\exp \left\{-\beta \int_{0}^{t} V(u) d u\right\} F(V(t), t)
$$

where, for $\alpha \geq 0, \beta \geq-\kappa^{2} / 2 \sigma_{v}^{2}$, we have

$$
F(V(t), t)=\exp \left\{-(\kappa \theta) \phi_{\alpha, \beta}(t)\right\} \exp \left\{-V(0) \psi_{\alpha, \beta}(t)\right\}
$$

as a solution to the Cauchy problem

$$
\begin{gathered}
\frac{\partial F}{\partial t}(v, t)+\frac{1}{2} \sigma_{v}^{2} v \frac{\partial^{2} F}{\partial v^{2}}+\kappa(\theta-v) \frac{\partial F}{\partial v}=\beta v F, \\
F(v, T)=e^{-\alpha v} .
\end{gathered}
$$

The coefficients $\phi_{\alpha, \beta}(t), \psi_{\alpha, \beta}(t)$ satisfy the Riccati ODE

$$
\begin{gathered}
\psi^{\prime}(t)=\frac{\sigma_{v}^{2}}{2} \psi^{2}(t)+\kappa \psi(t)-\beta, \\
-\phi^{\prime}(t)=\psi(t), \\
\psi(T)=\alpha, \phi(T)=0 .
\end{gathered}
$$

The solution for $\beta>-\kappa^{2} / 2 \sigma_{v}^{2}$ is

$$
\begin{gathered}
\eta=\sqrt{\kappa^{2}+2 \sigma_{v}^{2} \beta}, \\
\phi_{\alpha, \beta}(t)=-\frac{2}{\sigma_{v}^{2}} \ln \left(\frac{2 \eta \exp \{(1 / 2)(T-t)(\eta+\kappa)\}}{\sigma_{v}^{2} \alpha\left(e^{\eta(T-t)}-1\right)+\eta-\kappa+e^{\eta(T-t)}(\eta+\kappa)}\right), \\
\psi_{\alpha, \beta}(t)=\frac{\alpha\left(\eta+\kappa+e^{\eta(T-t)}(\eta-\kappa)\right)+2 \beta\left(e^{\eta(T-t)}-1\right)}{\sigma_{v}^{2} \alpha\left(e^{\eta(T-t)}-1\right)+\eta-\kappa+e^{\eta(T-t)}(\eta+\kappa)},
\end{gathered}
$$


and for $\beta=-\kappa^{2} / 2 \sigma_{v}^{2}$ is

$$
\begin{gathered}
\phi_{\alpha, \beta}(t)=-\frac{2}{\sigma_{v}^{2}} \ln \left(\frac{2 \exp \{(1 / 2) \kappa(T-t)\}}{\alpha \sigma_{v}^{2}(T-t)+2+\kappa^{2}(T-t)}\right), \\
\psi_{\alpha, \beta}(t)=\frac{2 \alpha \sigma_{v}^{2}-\kappa \alpha \sigma_{v}^{2}(T-t)-\kappa^{2}(T-t)}{\sigma_{v}^{2}\left(\alpha \sigma_{v}^{2}(T-t)+2+\kappa^{2}(T-t)\right)} .
\end{gathered}
$$

Applying Itôs formula to (3.12) we see that

$$
d M(t)=e^{\left\{-\beta \int_{0}^{t} V(u) d u\right\}} \frac{\partial F}{\partial v} \sigma_{v} \sqrt{V(t)} d W_{1}(t)
$$

which shows that $M(\cdot)$ is a local martingale. Notice that from (3.12) and (3.13), we see that the local martingale $M(\cdot)$ is nonnegative by Fatou's lemma is a supermartingale. Hence

$$
E[M(T)] \leq M(0)=F(V(0), 0)<\infty
$$

as required.

Remark 3.2. With $\beta \geq 0$, (3.19) will actually hold with equality as a result of the FeynmanKac formula.

Corollary 3.3. For $|\lambda| \leq \kappa / \sigma_{v}$,

$$
E\left[L_{1}(T)\right]=1,
$$

and hence the postulated ELMM exists.

Proof. Consider $\alpha=0,0>\beta \geq-\kappa^{2} / 2 \sigma_{v}^{2}$. Direct substitution in (3.19) gives

$$
E\left[e^{\left\{-\beta \int_{0}^{t} V(u) d u\right\}}\right]<\infty,
$$

and hence, for $|\lambda| \leq \kappa / \sigma$ we have

$$
E\left[\exp \left\{\frac{1}{2} \lambda^{2} \int_{0}^{t} V(u) d u\right\}\right]<\infty
$$

and by Novikov's condition (3.20) is proved.

Proposition 3.4. For all $0<\lambda<\infty$,

$$
E\left[L_{1}(T)\right]=1 .
$$

Proof. Rearrangement of the equation for $V(\cdot)$ gives

$$
\lambda \int_{0}^{T} \sqrt{V(u)} d W(u)=\frac{\lambda}{\sigma_{v}}\left(V(T)-V(0)+\int_{0}^{T} \kappa \theta d u-\int_{0}^{T} \kappa V(u) d u\right) .
$$


8 On changes of measure in stochastic volatility models

On substitution into $L_{1}(T)$ we find that

$$
\begin{aligned}
E\left[L_{1}(T)\right]= & \exp \left\{\frac{\lambda}{\sigma_{v}}(V(0)+\kappa \theta T)\right\} \\
& \times E\left[\exp \left\{-\left(\frac{\lambda}{\sigma_{v}}\right) V(T)-\left(\frac{\lambda \kappa}{\sigma_{v}}+\frac{1}{2} \lambda^{2}\right) \int_{0}^{T} V(u) d u\right\}\right] .
\end{aligned}
$$

Equation (3.23) follows via the results of Remark 3.2 with $\alpha=\lambda / \sigma_{v}, \beta=\left(\lambda \kappa / \sigma_{v}+(1 / 2) \lambda^{2}\right)$.

Combining Corollary 3.3 and Proposition 3.4 we have the following theorem.

Theorem 3.5. If $-\kappa / \sigma_{v} \leq \lambda<\infty$,

$$
E[L(T)]=E\left[\exp \left\{-\int_{0}^{T} \lambda \sqrt{V(u)} d W_{1}(u)-\frac{1}{2} \int_{0}^{T}(\lambda \sqrt{V(u)})^{2} d u\right\}\right]=1
$$

implying that an equivalent local martingale measure corresponding to the market price of volatility risk factor $\lambda \sqrt{V(t)}$ exists.

It should further be noted that the problem investigated in this section is essentially the same as that encountered in the term structure model of Cox et al. [5]. Hence our results regarding the Heston model will be equally applicable to the CIR model.

3.2. Strictly local martingale property. In this section we will analyze the conditions required to ensure that discounted stock prices under an ELMM $Q$ are true martingales, as opposed to strictly local martingales. As with the assumptions behind the existence of ELMMs, this is again another martingale property which is often unchecked in many finance models. The most relevant examples where this problem occurs include the HullWhite stochastic volatility model with positive correlation, as pointed out by Sin [30], as well as versions of the constant elasticity of variance model, as pointed out by Heath and Schweizer [16]. Whilst strictly local martingale discounted stock prices do not involve strict arbitrage opportunities per se (see Delbaen and Schachermayer [8]), they can create a multitude of problems, for example, causing an inability to price derivatives using expectations as well as other counter-intuitive "arbitrage" opportunities, including a failure of the put-call parity theorem. For a detailed discussion of these problems the reader is referred to Heath and Schweizer [16], and Cox and Hobson [4].

Our analysis of the strictly local martingale property is based on the observation that discounted stock prices under $Q$ are stochastic exponentials, and moreover that they are of the same form as that studied in Section 3.1. This close relationship between the two problems provides us with a means to apply the results derived in the previous section.

Theorem 3.6. Assume that an ELMM Q exists in the Heston stochastic volatility model. The discounted stock price is a Q-martingale if

$$
\kappa+\lambda \sigma_{v} \geq \sigma_{v} \rho
$$


Proof. We know from Section 3.1 that under $Q$ the volatility process has dynamics

$$
d V(t)=\kappa \theta d t-\left(\kappa+\lambda \sigma_{v}\right) V(t) d t+\sigma_{v} \sqrt{V(t)} d W_{1}^{Q}(t) .
$$

The discounted stock price $Z(\cdot)$ can be represented by

$$
Z(T)=\exp \left\{\rho \int_{0}^{T} \sqrt{V(t)} d W_{1}^{Q}(t)+\sqrt{1-\rho^{2}} \int_{0}^{T} \sqrt{V(t)} d W_{2}^{Q}(t)-\frac{1}{2} \int_{0}^{T} V(t) d t\right\} .
$$

By the conditioning argument used in Section 3.1 we have

$$
E[Z(T)]=E\left[\exp \left\{\rho \int_{0}^{T} \sqrt{V(t)} d W_{1}^{Q}(t)-\frac{1}{2} \rho^{2} \int_{0}^{T} V(t) d t\right\}\right]
$$

which on comparison with Theorem 3.5 (with a change on parameters since we are starting from the $Q$ dynamics) we find that the requirement for

$$
E[Z(T)]=1
$$

is met if

$$
\rho \leq \frac{\kappa+\lambda \sigma_{v}}{\sigma_{v}}
$$

\section{Markovian stochastic volatility models}

We will now extend our results and develop a partial-differential-equation-based theorem that is applicable to a broad class of stochastic volatility models. Following Hoffman et al. [19], a general class of Markovian stochastic volatility framework can be given by

$$
\begin{gathered}
d X(t)=X(t)\left(\mu(t, X(t), V(t)) d t+\sigma_{1}(t, X(t), V(t)) d W_{1}(t)\right. \\
\left.+\sigma_{2}(t, X(t), V(t)) d W_{2}(t)\right), \\
d V(t)=\varsigma(t, X(t), V(t)) d t+\eta(t, X(t), V(t)) d W_{1}(t),
\end{gathered}
$$

with $X(0), V(0)>0$. This class nests the core of the Brownian-motion-based stochastic volatility models encompassed in the literature, including the models of Heston [18], Hull and White [20], Lewis [23]. We will also assume the existence of a savings account process

$$
B(t)=B(0) e^{r t} ; \quad B(0)=1
$$

with constant risk-free rate $r$.

For the model to admit an ELMM, it is clear that the first assumption is that the market price of risk processes $\gamma_{1}(t, X(t), V(t)), \gamma_{2}(t, X(t), V(t))$ satisfies

$$
\begin{aligned}
\mu(t, X(t), V(t))-r= & \gamma_{1}(t, X(t), V(t)) \sigma_{1}(t, X(t), V(t)) \\
& +\gamma_{2}(t, X(t), V(t)) \sigma_{2}(t, X(t), V(t))
\end{aligned}
$$


as this is required if discounted stock prices are to be local martingales. As we have an incomplete market, a second equation is not available to determine uniquely the market prices of risk processes.

In applications one often specifies a certain class of ELMMs of interest. We consider

$$
\vartheta(t, X(t), V(t))=\eta(t, X(t), V(t)) \gamma_{1}(t, X(t), V(t))
$$

for some process $\vartheta(\cdot, X(\cdot), V(\cdot))$ specified to identify the particular ELMM one wants to use for derivative pricing. See, for example, Henderson [17] for some comparisons and extensive references.

Our main result follows.

Theorem 4.1. Consider a stochastic volatility model (4.1) with market price of risk process $\gamma_{1}(\cdot), \gamma_{2}(\cdot)$ satisfying (4.4), (4.5). Set

$$
Y(t)=\left[\begin{array}{llll}
\gamma_{1}(t) & \gamma_{2}(t) & X(t) & V(t)
\end{array}\right]^{\prime}
$$

with

$$
\begin{gathered}
d Y(t)=b(t, Y(t)) d t+s(t, Y(t)) \cdot d W(t), \\
a(t, y)=s(t, y) \cdot s(t, y)^{\prime} .
\end{gathered}
$$

Also assume that

$$
P\left(\int_{0}^{T}\|Y(t)\|^{2} d t<\infty\right)=1
$$

Suppose there exists a positive $v(t, y)$ such that

$$
v(t, y) \subset C\left([0, T] \times R^{d}\right) \cap C^{1,2}\left((0, T) \times R^{d}\right)
$$

which is a solution to the Cauchy problem

$$
\frac{\partial v}{\partial t}+\sum_{i=1}^{4} b_{i}(t, y) \frac{\partial v}{\partial y_{i}}+\frac{1}{2} \sum_{i, j=1}^{4} a_{i, j}(t, y) \frac{\partial^{2} v}{\partial y_{i} \partial y_{j}}=c(t, y) v(t, y)
$$

with

$$
c(u, Y(u))=-\frac{1}{2} \sum_{i=1}^{2} Y_{i}^{2}(u)
$$

and boundary condition

$$
v(T, y)=1 \text {. }
$$


Then the stochastic exponential

$$
\xi_{0}^{t}(Y(u))=\exp \left\{\int_{0}^{t} \sum_{i=1}^{k} Y_{i}(u) d W_{i}(u)-\frac{1}{2} \int_{0}^{t} \sum_{i=1}^{k} Y_{i}^{2}(u) d u\right\}
$$

is a true martingale, and hence an associated equivalent (local) martingale measure exists. Proof. Consider the process $\{M(t)\}_{0 \leq t \leq T}$ with

$$
M(t)=\exp \left\{-\int_{0}^{t} c(u, Y(u)) d u\right\} v(t, Y(t))
$$

From Itôs formula and (4.8) we see that

$$
d M(t)=\sum_{i=1}^{4} e^{\left\{-\int_{0}^{t} c(u, Y(u)) d u\right\}} s_{i}(t, Y(t)) \frac{\partial v}{\partial y_{i}} d W_{i}(t),
$$

which shows that $M(t)$ is a local martingale. Furthermore we see that

$$
M(t)=\exp \left\{-\int_{0}^{t} c(u, Y(u)) d u\right\} v(t, Y(t))>0,
$$

for $0 \leq t \leq T$. Hence by Fatou's lemma it is a supermartingale

$$
E[M(T)] \leq M(0),
$$

which, on substitution, gives

$$
E\left[e^{-\int_{0}^{T} c(u, Y(u)) d u}\right]=E\left[e^{(1 / 2) \int_{0}^{t} \sum_{i=1}^{k} Y_{i}^{2}(u) d u}\right] \leq v(0, Y(0))<\infty,
$$

and hence by Novikov's condition $\xi_{0}^{t}(Y(\cdot))$ is a true martingale.

This theorem allows us to check the equivalence of $P$ and a particular class $Q$ of possible ELMMs. If the selected $Q$ was in fact the only possible choice then of course we can arrive at a sharper statement. The reader is referred to Friedman [13] for results stating whether solutions exist for a particular Cauchy problem (4.11). Furthermore in applications a simple numerical experiment could quite easily suggest whether a solution exists or not.

It should also be noted that a simple extension of Theorem 4.1 is equally applicable to the problem of ensuring that a particular change of measure such as a change of numeraire (Geman et al. [14]) is internally consistent or not, and is of particular relevance to models of exchange rates (Delbaen and Schachermayer [9]). 


\section{References}

[1] C. Ball and A. Roma, Stochastic volatility option pricing, Journal of Financial and Quantitative Analysis 29 (1994), no. 4, 584-607.

[2] B. Bibby and M. Sørensen, A hyperbolic diffusion model for stock prices, Finance and Stochastics 1 (1997), no. 1, 25-41.

[3] M. Chernov and E. Ghysels, A study towards a unified approach to the joint estimation of objective and risk neutral measures for the purpose of options valuation, Journal of Financial Economics 56 (2000), no. 3, 407-458.

[4] A. M. G. Cox and D. G. Hobson, Local martingales, bubbles and option prices, Finance and Stochastics 9 (2005), no. 4, 477-492.

[5] J. C. Cox, J. E. Ingersoll Jr., and S. A. Ross, A theory of the term structure of interest rates, Econometrica 53 (1985), no. 2, 385-408.

[6] Q. Dai and K. Singleton, Specification analysis of affine term structure models, Journal of Finance 55 (2000), no. 5, 1943-1978.

[7] F. Delbaen and W. Schachermayer, A general version of the fundamental theorem of asset pricing, Mathematische Annalen 300 (1994), no. 3, 463-520.

[8] - The existence of absolutely continuous local martingale measures, The Annals of Applied Probability 5 (1995), no. 4, 926-945.

[9] - The no-arbitrage property under a change of numéraire, Stochastics and Stochastics Reports 53 (1995), no. 3-4, 213-226.

[10] G. Duffee, Term premia and interest rate forecasts in affine models, Journal of Finance 57 (2002), no. $1,405-443$.

[11] W. Feller, Two singular diffusion problems, Annals of Mathematics. Second Series 54 (1951), 173182.

[12] R. Frey, Derivative asset analysis in models with level-dependent and stochastic volatility, CWI Quarterly 10 (1997), no. 1, 1-34.

[13] A. Friedman, Partial Differential Equations of Parabolic Type, Prentice-Hall, New Jersey, 1964.

[14] H. Geman, N. El Karoui, and J.-C. Rochet, Changes of numéraire, changes of probability measure and option pricing, Journal of Applied Probability 32 (1995), no. 2, 443-458.

[15] D. Heath, E. Platen, and M. Schweizer, A comparison of two quadratic approaches to hedging in incomplete markets, Mathematical Finance 11 (2001), no. 4, 385-413.

[16] D. Heath and M. Schweizer, Martingales versus PDEs in finance: an equivalence result with examples, Journal of Applied Probability 37 (2000), no. 4, 947-957.

[17] V. Henderson, Analytical comparisons of option prices in stochastic volatility models, Mathematical Finance 15 (2005), no. 1, 49-59.

[18] S. Heston, A closed form solution for options with stochastic volatility with applications to bond and currency options, Review of Financial Studies 6 (1993), no. 2, 327-344.

[19] N. Hoffman, E. Platen, and M. Schweizer, Option pricing under incompleteness and stochastic volatility, Mathematical Finance 2 (1992), 153-187.

[20] J. Hull and A. White, The pricing of options on assets with stochastic volatilities, Journal of Finance 42 (1987), no. 2, 281-300.

[21] G. Johnson and L. L. Helms, Class D supermartingales, Bulletin of the American Mathematical Society 69 (1963), 59-62.

[22] I. Karatzas and S. E. Shreve, Brownian Motion and Stochastic Calculus, Graduate Texts in Mathematics, vol. 113, Springer, New York, 1988.

[23] A. L. Lewis, Option Valuation under Stochastic Volatility, Finance Press, California, 2000.

[24] J. Pitman and M. Yor, A decomposition of Bessel bridges, Zeitschrift für Wahrscheinlichkeitstheorie und Verwandte Gebiete 59 (1982), no. 4, 425-457.

[25] D. Revuz and M. Yor, Continuous Martingales and Brownian Motion, Fundamental Principles of Mathematical Sciences, vol. 293, Springer, Berlin, 1991. 
[26] L. C. G. Rogers and D. Williams, Diffusions, Markov Processes, and Martingales. Vol. 2, 2nd ed., Cambridge Mathematical Library, Cambridge University Press, Cambridge, 2000, originally published by Wiley, Chichester, 1987.

[27] T. Rydberg, A note on the existence of equivalent martingale measures in a Markovian setting, Finance and Stochastics 1 (1997), no. 3, 251-257.

[28] T. H. Rydberg, Generalized hyperbolic diffusion processes with applications in finance, Mathematical Finance 9 (1999), no. 2, 183-201.

[29] R. Schöbel and J. Zhu, Stochastic volatility with an Ornstein-Uhlenbeck process: an extension, European Finance Review 3 (1999), no. 1, 23-46.

[30] C. A. Sin, Complications with stochastic volatility models, Advances in Applied Probability 30 (1998), no. 1, 256-268.

[31] E. Stein and J. Stein, Stock price distributions with stochastic volatility: an analytic approach, Review of Financial Studies 4 (1991), no. 4, 727-752.

[32] B. Wong and C. C. Heyde, On the martingale property of stochastic exponentials, Journal of Applied Probability 41 (2004), no. 3, 654-664.

Bernard Wong: School of Actuarial Studies, University of New South Wales,

Sydney, NSW 2152, Australia

E-mail address: bernard.wong@unsw.edu.au

C. C. Heyde: Mathematical Sciences Institute, The Australian National University,

Canberra, ACT 0200, Australia

Current address: Department of Statistics, Columbia University, New York, NY 10027, USA

E-mail address: chris@maths.anu.edu.au 


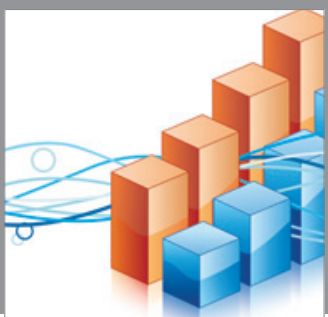

Advances in

Operations Research

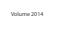

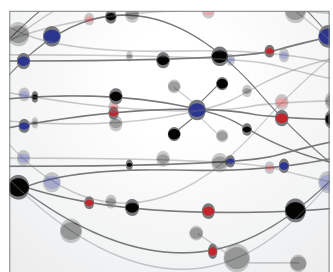

\section{The Scientific} World Journal
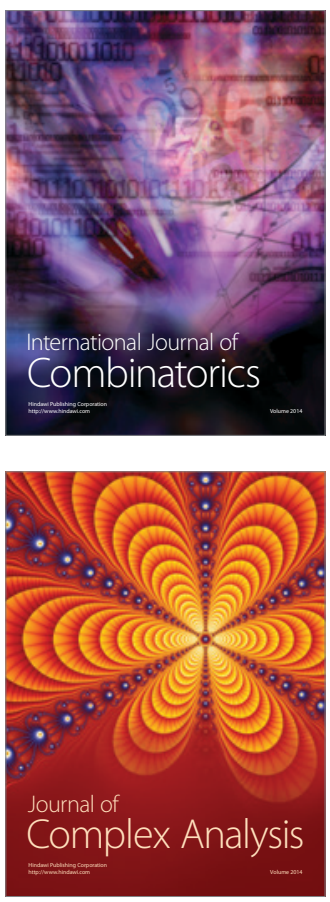

International Journal of

Mathematics and

Mathematical

Sciences
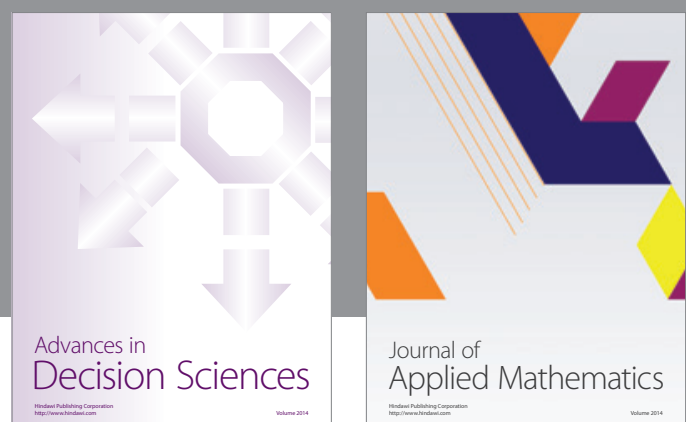

Journal of

Applied Mathematics
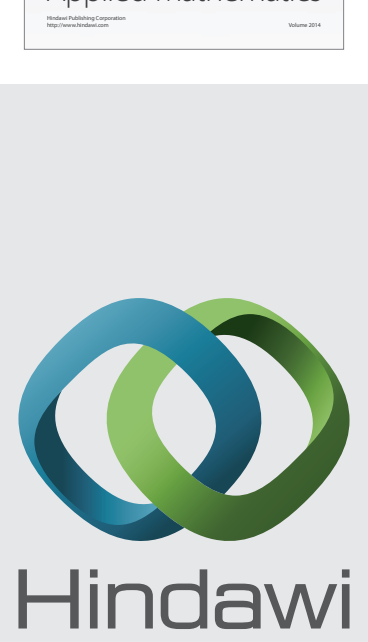

Submit your manuscripts at http://www.hindawi.com
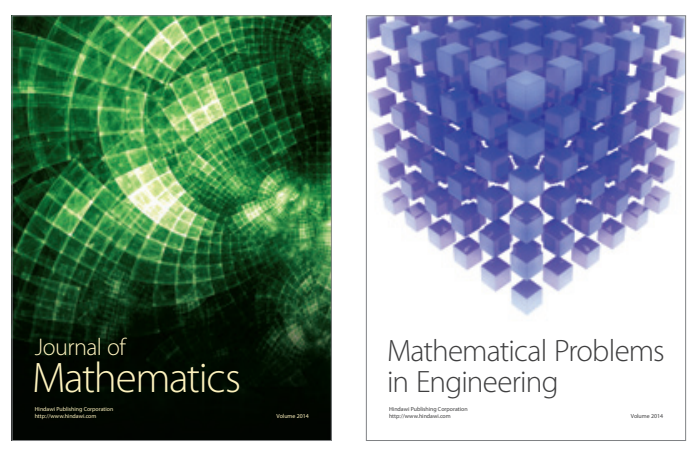

Mathematical Problems in Engineering
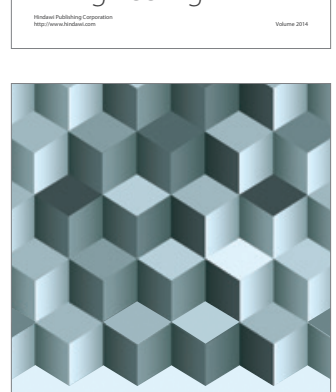

Journal of

Function Spaces
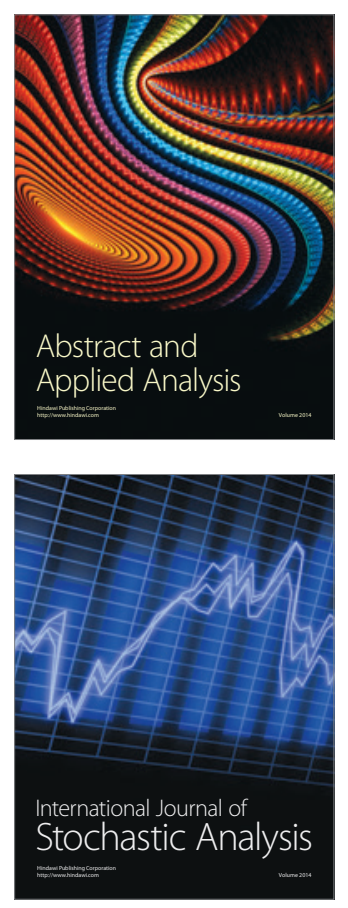

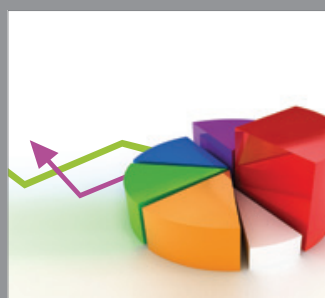

ournal of

Probability and Statistics

Promensencen
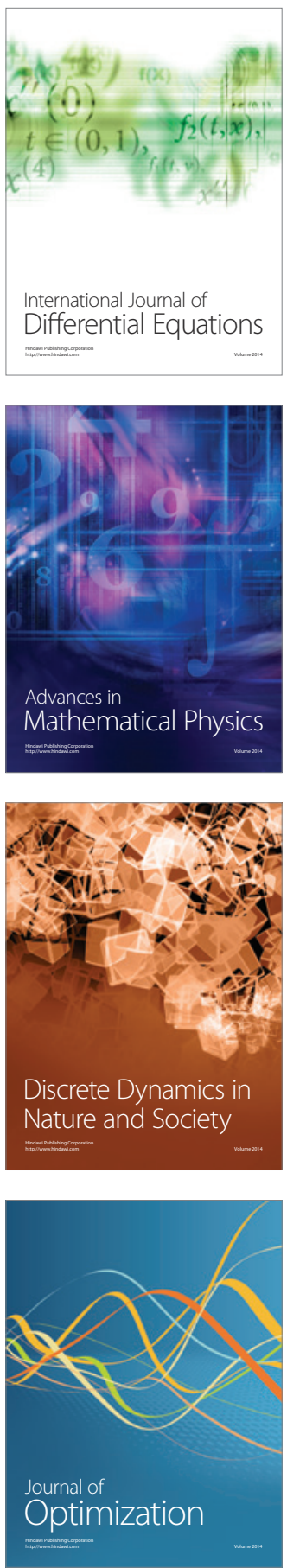